\title{
Slow dynamics about the glass transition as explored by muon spin relaxation spectroscopy
}

\author{
C. Cabrillo, ${ }^{1}$ F. J. Bermejo, ${ }^{1,2}$ and S. F. J. Cox ${ }^{3}$ \\ ${ }^{1}$ Instituto de Estructura de la Materia, Consejo Superior de Investigaciones Cientificas, Serrano 123, E-28006 Madrid, Spain \\ ${ }^{2}$ Department of Electricity and Electronics, Faculty Science, University of the Basque Country, P.O. Box 644, E-48080 Bilbao, Spain \\ ${ }^{3}$ Rutherford Appleton Laboratory, Chilton, Didcot Oxon OX11 OQX, United Kingdom
}

(Received 30 October 2002; published 6 May 2003)

\begin{abstract}
The glass transition of a molecular glass former is monitored by means of measurements of the spin relaxation rates of implanted muons. The data measured under transverse fields upon heating the material from the glass phase are consistent with the onset of ergodicity-restoring motions some $22 \mathrm{~K}$ above the calorimetric glass transition. The results show that the technique can be fruitfully exploited to assess the presence of criticality within the supercooled liquid at microsecond scales.
\end{abstract}

DOI: 10.1103/PhysRevB.67.184201

PACS number(s): 63.50. $+\mathrm{x}, 61.43 . \mathrm{Fs}$

Our present view on how a glassy solid melts into the supercooled liquid (SCL) state portrays it as due to the onset of diffusive molecular motions as the glass transition temperature $T_{\mathrm{g}}$ is crossed from below. Such motions are often explored at frequencies spanning from $10^{-9} \mathrm{~Hz}$ up to a few $\mathrm{THz}$ by dielectric and NMR spectroscopies and by radiation scattering techniques that provide microscopic information. The temperature dependence of the main relaxation time (the " $\alpha$ peak") of the dielectric function often departs from thermally activated (Arrhenius) behavior as one approaches $T_{\mathrm{g}}$ as also does the macroscopic viscosity. Phenomenologically this is understood on the grounds of "cooperatively rearranging regions" " that increase in size with decreasing temperature. In contrast, a dynamic signature of the onset of liquid flow motions resulting from thermal activation of density fluctuations was predicted by kinetic theory ${ }^{2}$ to lie at a characteristic temperature usually referred to as $T_{\mathrm{c}}$ a few tens of kelvins above $T_{\mathrm{g}}$. While simulation results on ideal systems lend support to some of the predictions, ${ }^{3}$ their experimental scrutiny is riddled by difficulties that result from the intricacies of particle motions present in laboratory glass formers which may hide, at least in part, the sought dynamic phenomenon. In fact, the huge viscosity of a deeply SCL forces a tagged particle within a "cooperatively rearranging region" 1 to move by concerted rearrangements with its neighbors, usually involving motions of different nature (i.e., rototranslational motions, molecular deformational movements, etc.).

Experimental efforts to assess the physical soundness of $T_{\text {c }}$ require sophisticated data treatments ${ }^{4}$ to unveil hints of critical behavior. The difficulties arise from the existence in actual materials of dynamic phenomena that are strongly coupled to the density fluctuations and therefore their effect needs to be isolated from the measured response. Such motions may exhibit distinct dependences with temperature from that followed by translational mass diffusion and may therefore obscure any sign of criticality at $T_{\mathrm{c}}$ as it has now been recognized. ${ }^{5}$

Here we report on the glassy dynamics for times $\approx 0.01 \mu \mathrm{s} \leqslant t \leqslant 10 \mu \mathrm{s}$ which may shed light on the dilemma referred to above. Our time window spans a largely uncharted territory with time scales about $T_{\mathrm{g}}$, well below those sampled by macroscopic measurements, but significantly above those of studies using depolarized light ${ }^{4}$ or neutron ${ }^{2}$ scattering (i.e., $10^{-9}-10^{-12} \mathrm{~s}$ ) that are sensitive to both inter- and intramolecular motions. Our technique consists in the measurement of the temperature dependence of the relaxation rates of implanted muons onto a sample, namely, ethanol, ${ }^{6}$ for which its dynamics about $T_{\mathrm{g}}$ is known in detail. Because of the small muon mass, its motions once implanted will be driven by the excitations of its microscopic environment to which it is strongly coupled. The latter depends upon known radiochemical details. Two kinds of muon species are here expected. Some muons will capture an electron, leading to muonium formation $(\mathrm{Mu})$, while others will stick to ethanol molecules near the oxygen attracted by its electronegativity (EthHO-Mu ${ }^{+}$) or even substitute hydrogen (EthOMu), both cases being diamagnetic (i.e., closed shell) in character.

The measurements were carried out using the muon spin resonance $(\mu \mathrm{SR})$ spectrometer at ISIS. The glass sample was prepared by a deep quench into LN2 previous to insertion into a cryostat kept well below $T_{\mathrm{g}}=96 \mathrm{~K}$. Spectra of the glassy sample under longitudinal (LF) and transverse (TF) fields were then measured from $5 \mathrm{~K}$ up to temperatures corresponding to the normal liquid range $(200 \mathrm{~K})$. A subsequent slow cooling run followed in order to provide measurements of the sample in its ordered monoclinic crystal state $\left(T_{m}\right.$ $=159 \mathrm{~K})$. Because of the characteristics of the ISIS instrument, one expects either a relaxation curve of the initially $100 \%$ polarized muon spins towards the thermal polarization values for the LF experiments or a damping of the muon precession signals for the TF case. The relaxation towards equilibrium of the LF setup is driven by the $J\left(\omega_{0}\right)$ spectral density of the local magnetic field fluctuations at the Larmor frequency for the muon states (or, for muonium, at various transition frequencies of the hyperfine-coupled spin states). In contrast, the TF arrangement is also sensitive to static or "frozen-in" spatial fluctuations of the local magnetic field since they induce dephasing among the spin precession of muons located at different sites. In other words, the relaxation is also driven by a zero-frequency term $J(0)$. The TF data taken at a pulsed muon source such as ISIS provide access to details of the dynamics of diamagnetic species only while the LF data sense also the paramagnetic muonium. The spectra yield relaxation rates $\lambda_{1,2}(T)=T_{1}^{-1}(\mathrm{LF}), T_{2}^{-1}(\mathrm{TF})$ and their corresponding amplitudes. 


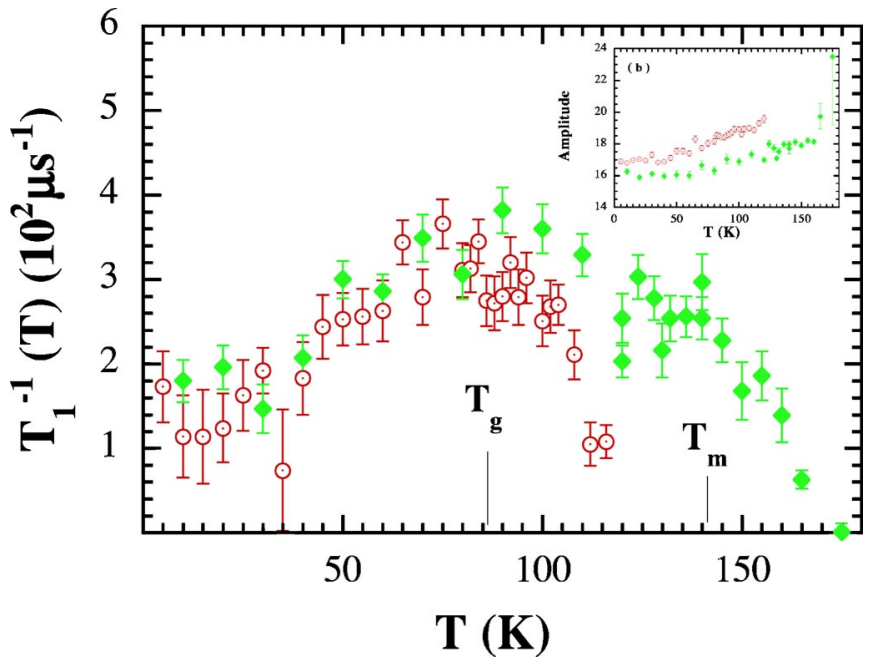

FIG. 1. (Color online) Longitudinal relaxation rates and relaxation amplitudes (inset) for the slowest relaxation appearing in spectra for an applied field of $0.1 \mathrm{kG}$. Data for the monoclinic crystal are depicted by solid symbols. Those for the glasssupercooled and normal liquid are shown as open circles.

Let us first consider the LF data. For fields of $100 \mathrm{G}$ the relaxation curves show two exponential components with well-separated decay times of orders of $100 \mu \mathrm{s}$ and $1 \mu \mathrm{s}$, respectively. Such two-component relaxation is consistent with the presence of two kinds of muon species: the paramagnetic $\mathrm{Mu}$ that has a strong response to the local magnetic fields with response times short enough to follow the diffusion among the interstitial sites and the muons fixed to the diamagnetic complexes (EtHO- $\mathrm{Mu}^{+}$and/or EtOMu) that relax at a considerably slower rate. This picture was ascertained by studying the field dependence of the relaxation parameters which show a maximum at $\approx 35 \mathrm{G}$ consistent with the presence of a diamagnetic complex plus a higherfield $(\mathbf{B}>2 \mathrm{kG})$ component attributable to atomic muonium. The fast component shows a weak dependence with temperature and physical state of the sample and therefore does not seem to provide relevant information for our study. The slower relaxation shows, however, a marked dependence with temperature as well as whether the sample is in its crystal or glass-liquid forms. The most significant results from such measurements are shown in Fig. 1. The $T_{1}^{-1}$ rates show a nonmonotonic temperature dependence and a hysteretic difference between glass and crystal above $\approx 50 \mathrm{~K}$. The rates for the latter are always larger than those for the glass and SCL and go through a broad maximum about $90 \mathrm{~K}$ whereas those for the glass peak at a somewhat lower temperature $(\approx 75 \mathrm{~K})$ and decrease strongly above $T_{\mathrm{g}}$. NMR relaxation rates $^{7}$ for the glass and SCL show a broad maximum at $\approx 83 \mathrm{~K}$ with Arrhenius parameters giving an activation energy as low as $1.9 \mathrm{~kJ} \mathrm{~mol}^{-1}$ and a preexponential term of $9.8 \times 10^{-9} \mathrm{~s}$. Data for the crystal show a maximum at $146 \mathrm{~K}$ arising from methyl group reorientations having an activation energy of $12.6 \mathrm{~kJ} \mathrm{~mol}^{-1}$ and a preexponential term of $3.3 \times 10^{-13} \mathrm{~s}$, while both $\mathrm{CH}_{2}$ and $\mathrm{OH}$ groups are stationary. Such relaxation due to the methyl group is well outside our frequency window. In contrast, the close temperatures where maxima for the glass appears in both $\mu$ SR and NMR measurements as well as the activation parameters that bring the correlation time into the microsecond range suggest that the same whole-molecule motions causing NMR relaxation should contribute to our observed $T_{1}^{-1}$ rate. The relaxation amplitude smoothly increases with temperature from about $50 \mathrm{~K}$ up to $120 \mathrm{~K}$ where the stability limit of the SCL is then reached and the sample crystallizes into its ordered form. The full signal amplitude is attained within the normal liquid range $(T \geqslant 175 \mathrm{~K})$ where the muon spins in the diamagnetic states cannot follow the dynamic phenomena (extreme narrowing).

The $T_{1}^{-1}$ rates show a nonmonotonic temperature dependence and a hysteretic difference between glass and crystal above $\approx 50 \mathrm{~K}$. Those for the latter are always larger than those for the glass and SCL and go through a broad maximum about $90 \mathrm{~K}$ whereas those for the glass peak at $(\approx 75 \mathrm{~K})$ and decrease strongly above $T_{\mathrm{g}}$. NMR relaxation rates $^{7}$ for the glass and SCL show a broad maximum at $\approx 83 \mathrm{~K}$ with an activation energy as low as $1.9 \mathrm{~kJ} \mathrm{~mol}^{-1}$ and a preexponential term of $9.8 \times 10^{-9} \mathrm{~s}$. Data for the crystal show a maximum at $146 \mathrm{~K}$ assigned to methyl group reorientations having an activation energy of $12.6 \mathrm{~kJ} \mathrm{~mol}^{-1}$ and a preexponential term of $3.3 \times 10^{-13} \mathrm{~s}$, while both $\mathrm{CH}_{2}$ and $\mathrm{OH}$ groups are stationary. Such figures show that such relaxation is well outside our frequency window. In contrast, the close temperatures where maxima for the glass appears in both $\mu$ SR and NMR measurements as well as the activation parameters that bring the correlation time into the microsecond range suggest that the same motions causing NMR relaxation should contribute to our observed $T_{1}^{-1}$ rate.

Transverse field measurements are sensitive to static spatial disorder and therefore can be compared to the second moment of the proton NMR line, ${ }^{7} M^{2}(T)$. The rigid-lattice value for the crystal ${ }^{7}$ is some $8 \mathrm{G}^{2}$ larger than the glass below $80 \mathrm{~K}$. Such a difference is also sensed by our $T_{2}^{-1}$ data down to $10 \mathrm{~K}$. The reduction of $M^{2}(T)$ or $T_{2}^{-1}$ with increasing temperature is known to arise from the onset of some molecular motions. These cannot be assigned to $\mathrm{CH}_{3}$ methyl group reorientations since our probe is fixed to the rigid part of the molecule (near the oxygen) which is about $r=3 \AA$ away from the methyl-group which makes direct dipolar couplings that are proportional to $r^{-6}$ have a small influence in the measured rates. Within the normal liquid state $(T$ $\geqslant 159 \mathrm{~K})$ motions become rather fast and only a slight dependence of $T_{2}^{-1}$ with temperature is found and, above 220 $\mathrm{K}$, go outside our instrument window. A sharp discontinuity of the $T_{2}$ rate is seen upon melting the crystal into normal liquid. In contrast, the glass data displays a far smoother behavior, showing no clear feature at $T_{\mathrm{g}}$ and evidencing a strong drop for higher temperatures.

So far our findings for both rates indicate that the temperature dependence of $\lambda(T)$ is governed by the onset of motions such as center-of-mass motions (c.m.), wholemolecule reorientations, or combinations of both. Our sample has an important advantage in this respect that helps to isolate the effects of rotations and c.m. motions to the observed rate. In fact, it can be prepared in the orientation- 


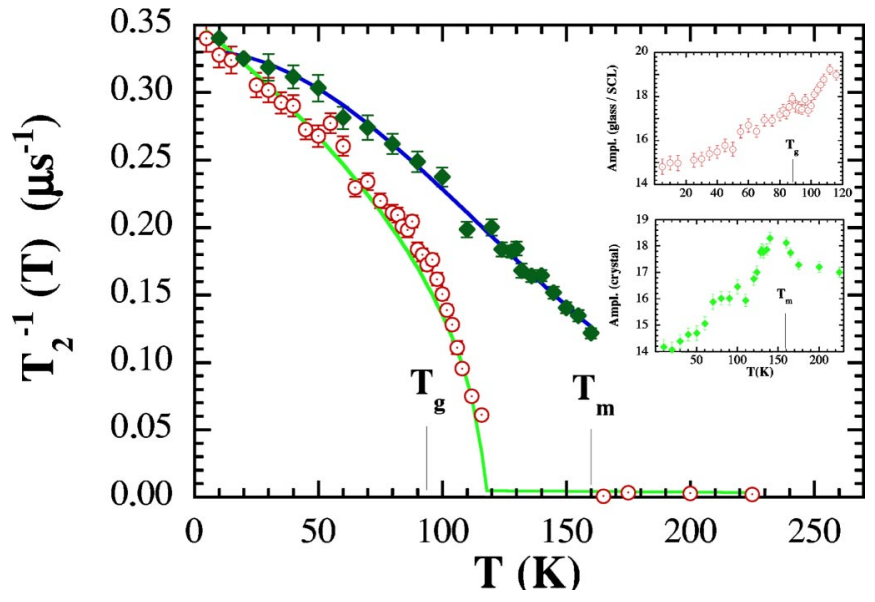

FIG. 2. (Color online) Transverse field relaxation rates. Open symbols represent data taken with the sample in glass and liquid forms. The measurements started with the glass sample at the lowest temperature, raising it subsequently up to the normal liquid ( $T$ $>159 \mathrm{~K}$ ) range and then cooling at a slow rate to form the ordered crystal (solid lozenges). The arrows in the abscissa depict the position of the calorimetric glass transition $T \mathrm{~g}$ and crystal melting $T_{\mathrm{m}}$ temperatures. The lines passing through the experimental points are model fits as described in the text. The insets show the temperature dependence of the relaxation amplitudes.

ally disordered glassy crystal ${ }^{6}$ form and it has enabled us to study the details of its molecular rotations about $T_{\mathrm{g}},{ }^{6}$ as well as to relate them to those taken place in the topologically disordered solid. The rotation frequencies were found to have a lower bound within the $\mathrm{GHz}$ range and also showed a drastic increase at $T_{\mathrm{g}}$. In consequence pure molecular rotations are not expected to contribute largely to our observed rate since their time scale lies outside the dynamic range of the present experiment.

For reasons explained above, the $T_{2}^{-1}$ rates monitor the development of a static component that parallels the behavior of the Lamb-Mössbauer (or Debye-Waller) factor as measured by various other spectroscopies. Figure 2 shows that above $50 \mathrm{~K}$, narrowing within the crystal and lowtemperature glass departs from the simple thermally activated behavior expected for the Debye-Waller factor of a harmonic sample (i.e., $\ln (\lambda(T)]$ significantly departs from a straight line). The crystal data can be accounted for if allow- ance is made for a $T^{2}$ term that represents the anharmonic vibrations of the molecular c.m. ${ }^{8}$ The resulting rate is thus $\lambda(T)=A_{\text {cryst }} \exp \left(-a T-b T^{2}\right)$, with temperature coefficients of $7.81 \times 10^{-4} \mathrm{~K}^{-1}$ and $-3.94 \times 10^{-6} \mathrm{~K}^{-2}$, respectively. A similar approach can only explain the glass data if the temperature is restricted up to $70 \mathrm{~K}$. One then needs some theoretically grounded form to explain the strong drop of $T_{2}^{-1}$ about and above $T_{\mathrm{g}}$. The whole range of glass-liquid data can be satisfactorily reproduced by the inclusion of a term $\propto \sqrt{\left|T-T_{c}\right| / T_{c}}$ following the prediction made by modecoupling theories (MCT's). ${ }^{2,9}$ Here $T_{\mathrm{c}}$ stands for the critical temperature referred to above. In such a way we now get

$$
\begin{gathered}
\lambda(T)=A_{\text {glass }} \sqrt{\left(T_{\text {red }} /(1-\gamma)\right.}+\zeta\left(T_{\text {red }}\right), \quad T \leqslant T_{c}, \\
\lambda(T)=\zeta\left(T_{\text {red }}\right), \quad T>T_{c},
\end{gathered}
$$

with

$$
T_{\text {red }}=\left|T-T_{c}\right| / T_{c}, \quad \zeta\left(T_{\text {red }}\right)=\alpha_{0}+\beta_{0} T_{\text {red }} .
$$

The approximation given by Eq. (1) satisfactorily accounts for the observations using a manageable number of parameters. Their values are $T_{\mathrm{c}}=118 \mathrm{~K}$, that is, $22 \mathrm{~K}$ above $T_{\mathrm{g}}$ - and the MCT exponent parameter $\gamma=0.61 .^{2}$ The hightemperature data are modeled by a mild, linear temperature dependence $\left(\alpha_{0}=0.0047\right.$ and $\left.\beta_{0}=-0.0012\right)$. The behavior of $T_{2}^{-1}$ about the glass transition is thus remarkably close to that predicted for the nonergodicity order parameter by the MCT (Ref. 2) for monoatomic liquids. Our previous studies on this sample ${ }^{6}$ enable us to interpret such a parameter as a measure of the progressive "freezing" of translational degrees of freedom during the structural arrest.

In summary, $\mu \mathrm{SR}$ serves to monitor the glassy dynamics at temperatures about $T_{\mathrm{g}}$ in a model-independent manner since the data treatment simply involves the fitting of exponential decays. The use of our particular sample has enabled us conduct a test of the MCT prediction free from ambiguities introduced by molecular degrees of freedom different from c.m. motions. The price to pay is the presence of a crystallization gap that prevented a detailed quantitative analysis of the behavior near the critical temperature. Other samples may perhaps be studied in the future using this promising technique.

We acknowledge support from DGICYT (Spain) Grant No. PB98-C02-01.
${ }^{1}$ G. Adam and J. H. Gibbs, J. Chem. Phys. 43, 139 (1965).

${ }^{2}$ W. Götze, J. Phys.: Condens. Matter 11, A1 (1999).

${ }^{3}$ W. Kob and H. C. Andersen, Phys. Rev. E 51, 4626 (1995); 52, 4134 (1995).

${ }^{4}$ H. Z. Cummins, J. Phys.: Condens. Matter 11, A95 (1999).

${ }^{5}$ R. Schilling, J. Phys.: Condens. Matter 12, 6311 (2000).

${ }^{6}$ A. Srinivasan et al., Phys. Rev. B 53, 8172 (1996); M. A. Ramos et al., Phys. Rev. Lett. 78, 82 (1997); M. Jiménez-Ruiz et al., Phys. Rev. B 59, 9155 (1999); C. Talón et al., ibid. 58, 745 (1998); A. Criado et al., ibid. 61, 8778 (2000); R. Fayos et al.,
Phys. Rev. Lett. 77, 3823 (1996); F. J. Bermejo et al., Phys. Rev. B 56, 11536 (1997); M. Jiménez-Ruiz et al., Phys. Rev. Lett. 83, 2757 (1999); C. Talón et al., Phys. Rev. B 66, 012201 (2002).

${ }^{7}$ T. Eguchi, G. Soda, and H. Chihara, Mol. Phys. 40, 681 (1980).

${ }^{8}$ A. A. Maradudin and P. A. Flinn, Phys. Rev. 129, 2529 (1963); H. E. Fischer et al., Phys. Rev. Lett. 82, 1193 (1999).

${ }^{9}$ T. Theenhaus et al., Phys. Rev. E 64, 051505 (2001); A. Winkler et al., ibid. 62, 8004 (2000); M. Letz, R. Schilling, and A. Latz, ibid. 62, 5173 (2000); R. Schilling, ibid. 65, 051206 (2002); S. H. Chong and W. Götze, ibid. 65, 041503 (2002). 